\title{
Analysis of China Underground Space Landscape Research Based on Data Visualization Technology
}

\author{
Qian Yao ${ }^{1, \mathrm{a}}$, Zhang Hua ${ }^{1, \mathrm{~b}}, \mathrm{Xu} \mathrm{Bin}^{2,3, \mathrm{c}^{*}}$, Wen $\mathrm{Cun}^{1, \mathrm{~d}}$ \\ ${ }^{1}$ Art school of Huzhou University, Huzhou, Zhejiang, China \\ ${ }^{2}$ China Architecture Design \& Research Group Beijing 100044 \\ ${ }^{3}$ Beijing University of Civil Engineering and Architecture Beijing 100044, China
}

\begin{abstract}
Based on the research from 2000 to 2020 in China Knowledge Resource Database (CNKI) as the data source, with the help of CiteSpace V and VOSviewer digital technology, a knowledge map is drawn on the number of articles, issuing organizations and keywords of underground space landscape in China. Visualization technology shows the relationship between the structure, evolution, and cooperation of underground space and landscape research, and intuitively shows the research hotspots and frontier dynamics of underground space landscape in China. The experiments results show that the underground space landscape was in its infancy before 2003, and then it began to develop rapidly. At present, underground space landscape research in China mainly focuses on hot spots such as landscape design, urban underground space, and public space. Through the visualized map analysis of the underground space landscape literature, it shows the use of new energy and new technology to create energy-saving underground buildings will become a new research trend.
\end{abstract}

\section{Introduction}

With the acceleration of urbanization, more and more problems accompanied it, such as urban land shortages, environmental pollution and so on. To deal with the problem of urban construction on the ground, people have begun to shift from the use of ground resources to the development of underground space. Compared with the above-ground space, underground space has more advantages in alleviating the pressure on the ground and improving the function of urban space. The rational development and utilization of underground space are bound to become a new development trend.

In 2016, $\mathrm{Hu}$ Lingxi reviewed the humanization of urban underground transportation space from the perspective of planning in "Research on the Humanization of Urban Underground Traffic Space", and proposed the principles of humanized design of urban underground transportation space and specific humanized design strategies $^{[1]}$. In 2019, Gao Weihao used the sunken plaza as the research object in the "Sinking Square Landscape Design from the Perspective of Urban Micro-Renewal"[2]. From the perspective of landscape, he researched and optimized the design of the space characteristics and landscape characteristics of the sunken plaza; In 2020, Liu Xiaojuan discussed the construction elements of underground space environmental landscape and the design focus of underground space environmental landscape in the "Deep and sub-deep underground space environmental characteristics and landscape construction",

ae-mail: Caroline0qy@163.com

be-mail: huayan.zhang@foxmail.com

*Corresponding author: cxubecobuild@163.com

de-mail: 18892646365@163.com in which the guiding ideology and design principles of environmental landscape planning can be used for reference in the underground deep and sub-deep space planning and design system ${ }^{[3]}$.

This article will analyze the current status of domestic research on the concept of underground space, discussing the research process of related scholars in this field. In view of the problems in the design process, relevant suggestions are made for future research trends, in order to provide references about development and construction for future research.

\section{Data sources and research methods}

\subsection{Source of data}

This article uses the China Knowledge Network (CNKI) database (journals-all journals). Based on the keywords of "underground space" and "landscape", the paper from 2000 to 2020 in the research were retrieved. The initial search data is 835 papers. This article mainly studies the current status of domestic underground space landscape research. Screened again based on the keyword "underground space landscape", and finally identified 249 articles as research objects, including 119 academic journals, 118 master's theses, 5 doctoral theses, and 7 conference papers. 


\section{2 research method}

In recent years, information visualization has become a hot spot in information management research, and scientific knowledge graphs have also become one of the hot research methods in bibliometrics. The current mainstream knowledge graph software tools include CiteSpace V, Thomson Data Analyzer, VOSviewer, BibExcel and Pajek. Relatively speaking, CiteSpace has better analysis functions and compatibility. It has advantages in analyzing the structure, law and distribution of invisible knowledge within data, revealing the dynamic development of disciplines and discovering the research frontiers of disciplines. VOSviewer can avoid the mutual coverage of important nodes and tags, and pay attention to the main information display of the data set. Therefore, VOSviewer and CiteSpace are selected for visual analysis. Through software analysis of the number of articles published in underground space landscape papers from 2000 to 2020, some knowledge graphs about the number of articles published, journal distribution and keywords are obtained. It reveals the development status and development process of underground space landscape. Save all selected documents-export references-choose NoteExpress and EndNote formats, and then import them into VOSviewer and CiteSpace respectively to generate related data maps. At the same time, download the number of papers, subject distribution, journals, keywords, publishing institutions, fund projects, highly cited papers and other information on CNKI.

\section{The volume of publications and journal distribution}

The data of the volume of papers can show the development of the field and the degree of attention from the side.

\subsection{Changes in the number of publications in the underground space landscape and the distribution of major journals}

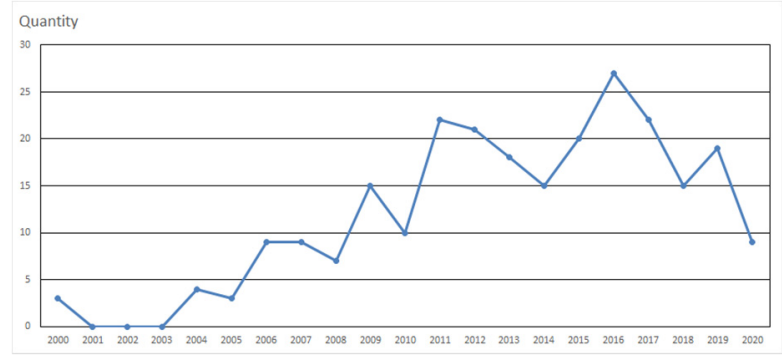

Figure 1 Changes in the amount of publications in underground space landscapes during 2000-2020

It can be seen from Figure 1 that from 2000 to 2020, the number of papers on underground space landscapes fluctuated in the early stage and showed a fluctuating downward trend in the later stage. It can be divided into three stages: From 2000 to 2003, the number of papers is small, and China has not started research on underground space. There was a volatile upward phase from 2004 to 2016 and a small peak was reached in 2011; it reached the highest peak in 2016; it was in a downward trend from 2016 to 2020 , and the relevant papers published in 2020 were only 9 articles. It can be seen that the research on underground space landscape design has gradually begun to pay attention in China. The subway construction in China has been in full swing in recent years. Since the National Land and Resources Bureau meeting in January 2017 emphasized the need for new urbanization to develop and utilize urban underground space, underground space landscape development has begun a new round of exploration in China.

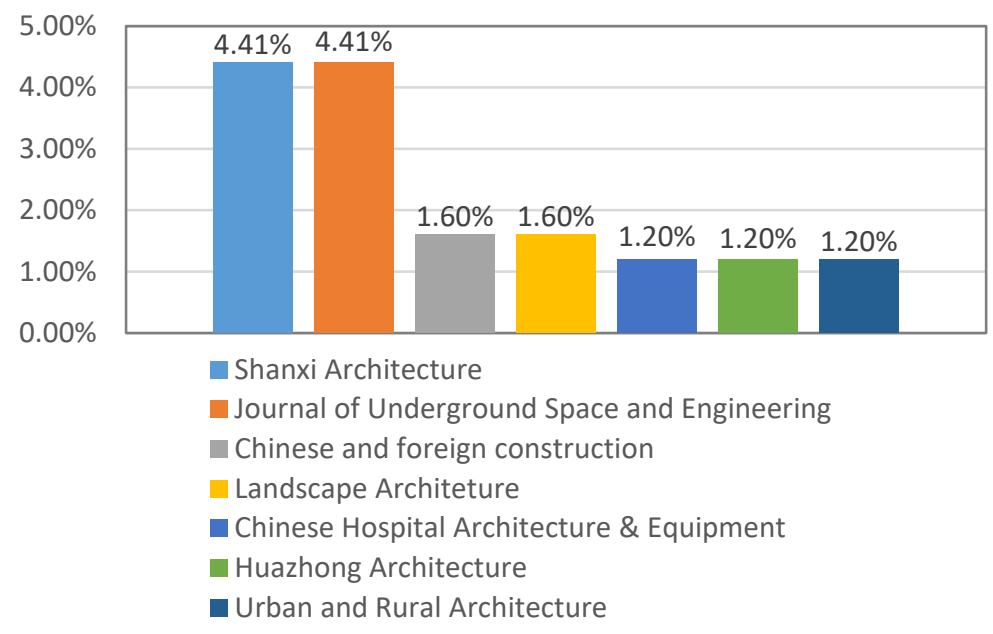

Figure 2 Analysis of the proportion of underground space landscape research journals from 2000 to 2020

It can be seen from Figure 2 that complied with other journals, "Shanxi Architecture" and "Journal of Underground Space and Engineering" have more articles. They each published 11 related articles and accounted for $4.41 \%$. However, most researches of these journals focus on architectural planning and other aspects, and less research on landscape. 


\section{The main content analysis}

\subsection{Highly cited papers}

The top 10 cited papers from 2000 to 2020 were selected from CNKI for analysis. The information about the top 5 papers is listed due to space limitation. See Figure 3 for details. The average number of citations of underground space landscape papers is 56 , and the average number of downloads is 2,785 . The main publishing institutions are Hunan University, Chongqing University, Tongji University, Central South University, etc. The main research contents of the top 10 cited papers focus on the design of underground commercial space, the design of the entrance of underground public space, the study of the underground space environment, and the design of sunken squares. Most of the emphasis is on the overall design of the underground space, and the landscape design is only partially combined when the overall research environment is built. It can be seen from this that the concentration of underground space environmental landscape design in the entire field is still not too high, and it is more researched as an embellishment within a large framework.

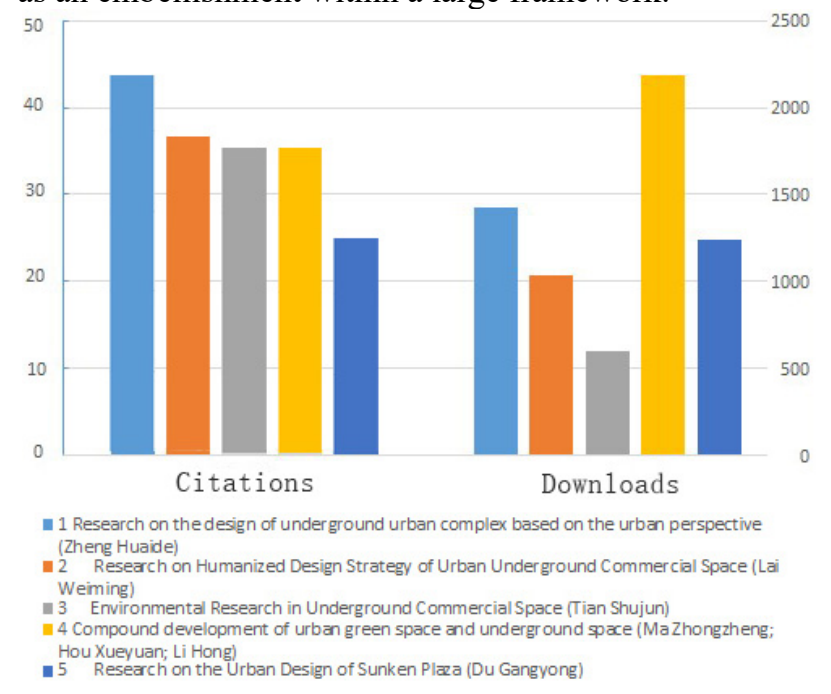

Figure 3 The top 5 papers cited in the underground space landscape field from 2000 to 2020

\subsection{Research hotspot analysis}

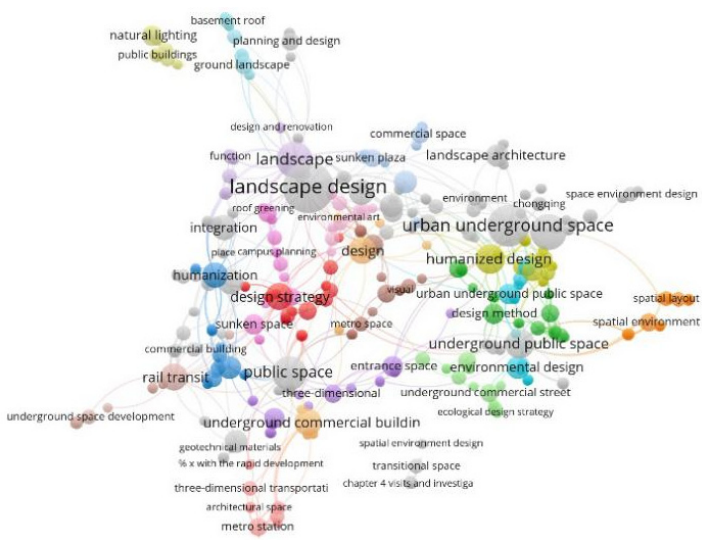

Figure 4 Analysis map of research hotspots in the field of underground space landscape from 2000 to 2020
In a certain period, the topics or perspectives studied by a group of documents are intrinsically connected and the number of which is large is called the research hotspot of this group of documents. Through keyword co-occurrence analysis, adjust the corresponding parameters, make highfrequency keywords more prominent and easy to read, and obtain the keyword co-occurrence knowledge map (Figure 4). The colored circular nodes in the figure represent keywords. The larger the circle, the higher the frequency of the keyword, and the larger the font, the greater its centrality. The connection between nodes is the degree of relevance between keywords. There are 260 keyword nodes in Figure 4. The distribution of words is relatively scattered. Most of the hot words are general words with a wider extension, which have not formed a relatively concentrated core hot area. It can be seen from the statistical high-frequency keyword map that "landscape design", "urban underground space", "underground commercial space", "public space" and "landscape" are all hot keywords with high frequency and belong to research hot topics.

\subsection{Analysis of Research Knowledge Evolution}

Different research hotspots are often derived from different periods. To show the evolution trend of research hotspots in this field more clearly, a time zone knowledge map of hotspot evolution has been drawn for the research on the ecological environment of underground space (Figure 5). The research is mainly divided into 4 stages.

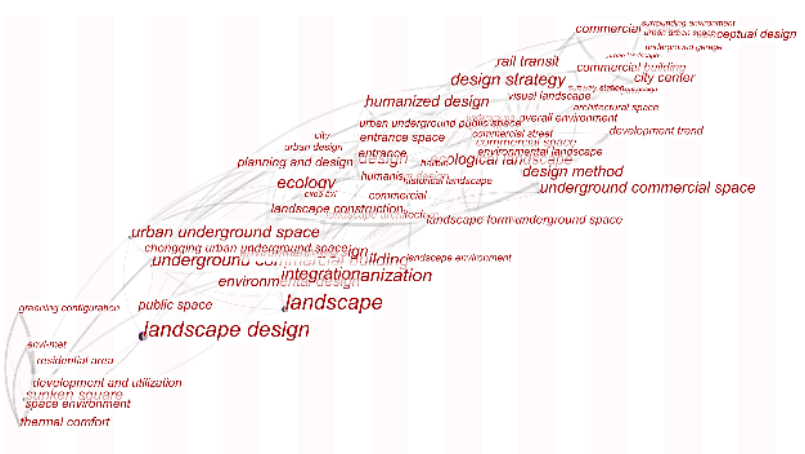

Figure 5 The time zone knowledge map of the evolution of underground space landscape research hotspots from 2000 to 2020

As is shown in the diagram, from 2000 to 2003, the research is in its initial stage. The main direction is the theoretical research on underground space. During this period, the literature discussion focus more on the functional development of underground space, and there are fewer literature related to the underground space landscape.

From 2004 to 2011, the development and utilization of underground space show a trend of further research. In 2005 , the work of building a conservation-oriented society was deployed, and the policy of putting conservation in the first place was put forward.$^{[4]}$ Under this background and the dual drive of rapid economic development, the research direction of underground space begin to develop in the direction of underground public space. From 2006 
to 2008 , more words such as "environmental design" and "landscape construction" appeared in the literature. At the same time, humanized design and historical landscape are more reflected in planning and design.

From 2012-2016, the study is in a prosperous and fluctuating period. The number of documents in this field reached the peak in 2016. While the research on "underground commercial space" and "sunken space landscape" is not diminishing, the entrances and exits of "metro space" and "underground space" have become new key research objects. "Integrated Rail Transit Design" and "Urban Integrated Design" Body design" and so on have gradually emerged. The combination of landscape design with different functions is more and more widely used in buildings.

During 2017-2020, the study have entered a period of decline. Before 2017, the research on the functions and forms of underground space utilization reached its peak. In 2017, the Bureau of Land and Resources issued a blue book on underground space development. Under the guidance of the new policy, the research hotspots have shifted to "design strategies" and "development trends" at this stage. Researchers begin to reflect on the development of underground space and landscape design in the past 20 years.

\section{Conclusion and suggestions}

Based on the document visualization software VOSviewer and CiteSpace, this paper analyzes the 249 effective documents in the field of underground space landscape research included in the CNKI database from 2000 to 2020 .

First, in terms of the number of studies, the amount of research literature in this field is low. From 2003 to 2016, there was a tortuous upward trend, but it has never published more than 30 articles in a year. There was a downward trend began in 2016, indicating that research on underground space landscape in China has entered a bottleneck period. Driven by policies, in-depth research on underground space landscape have become necessary.

Secondly, in terms of research content, the content span is relatively large and unable to form a relatively core hot spot. Moreover, the scope and depth of research direction involved in the literature are insufficient. There is not enough attention to the research of landscape design in underground space. For example, firstly, underground commercial spaces mostly focus on the commercial nature of commercial spaces without humanistic considerations. Secondly, while developing and using underground space, it neglects the protection of existing basic land, or blindly develops and utilizes it, resulting in a waste of resources.

Finally, in terms of scientific research institutions, the current literature researches are all of the scattershot type, and they have been researched in the identity of major universities or individuals. The interaction between teams is not strong. However, multi-domain and multi-type research modes are of great significance to increase the breadth and depth of research results. Enhancing the cooperative relationship between universities, enterprises and scientific research in this field is a powerful means to break through the current bottleneck period.
Through the visualized map analysis of the underground space landscape literature, and the promotion of the ecological concept, it becomes a new trend to use new energy and technology to create energy-saving underground buildings. Applying more natural elements to the design of the underground landscape space. ${ }^{[5]}$ This is also an exploration in the direction of the future underground space landscape design.

\section{Acknowledgements}

This research was Supported by "Beijing Advanced Innovation Center For Future Urban Design, Beijing University Of Civil Engineering And Architecture". The Study on Synergistic Technology Strategy for the Underground Block Space of Urban Railway Terminals Identified by Green Efficiency (W2018132-16) and The Study on interaction between Underground Space and Ecological Landscape in the View of Green Efficiency (X20020) by Beijing Advanced Innovation Center for Future Urban Design.

\section{References}

1. Hu Linxi. (2019). Research on Humanization of Urban Underground Traffic Space. Architecture and culture, 2016(6):128-131.

2. Gao Weihao. (2019). Landscape design of sunken plaza in urban micro-renewal perspective.

3. Liu Xiaojuan. (2019). Environmental Characteristics and Landscape Construction of Deep and Subdeep Underground Space.Jiangsu Architecture, 2019(03): 7-10.

4. Wen Jiabao. (2005). Attach great importance to strengthening leadership and speeding up the construction of an economical society. Land and Resources Newsletter, 14, 6-9.

5. Zhang Yi. (2019). The use of natural landscape elements in underground commercial spaces. Industrial Design, 000(011), 107-108. 000(011):107108. 